\title{
Periodic methanol masers in G9.62+0.20E (Research Note)
}

\author{
D. J. van der Walt ${ }^{1}$, J. P. Maswanganye ${ }^{1,2}$, S. Etoka ${ }^{3}$, S. Goedhart ${ }^{4,1}$, and S. P. van den Heever ${ }^{1}$ \\ ${ }^{1}$ Centre for Space Research, North-West University, 2790 Potchefstroom, South Africa \\ e-mail: johan.vanderwalt@nwu.ac.za \\ 2 Hartebeesthoek Radio Astronomy Observatory, 1740 Krugersdorp, South Africa \\ ${ }^{3}$ Hamburger Sternwarte, Universität Hamburg, 21029 Hamburg, Germany \\ ${ }^{4}$ SKA SA, The Park, Park Road, Pinelands, South Africa \\ Received 21 October 2015 / Accepted 17 January 2016
}

\section{ABSTRACT}

\begin{abstract}
A number of mechanisms for understanding the periodic class II methanol masers associated with some high-mass star forming regions have been proposed in the past. Two recent proposals by Parfenov \& Sobolev (2014, MNRAS, 444, 620) and Sanna et al. (2015, ApJ, 804, L2) have been presented in order to explain the periodic masers in sources with light curves similar to the methanol masers in G9.62+0.20E. We evaluate to what extent the proposals and models presented by these authors can explain the light curve of the methanol masers in G9.62+0.20E. It is argued that neither of the proposed mechanisms can reproduce the light curves of the methanol masers in G9.62+0.20E.
\end{abstract}

Key words. masers - ISM: molecules

\section{Introduction}

Recently, two papers (Parfenov \& Sobolev 2014; Sanna et al. 2015) have appeared in which explanations for the periodic behaviour of class II methanol masers in the high-mass star forming region G9.62+0.20E are presented. Parfenov \& Sobolev (2014) proposes that the flaring behaviour of periodic masers with light curves such as in G9.62+0.20E might be due to rotating spiral shocks in the gaps of circumbinary accretion disks around young binary stars. On the other hand, based on the results of a high-resolution astrometric study of $\mathrm{CH}_{3} \mathrm{OH}$, $\mathrm{H}_{2} \mathrm{O}$, and $\mathrm{OH}$ masers, as well as $7 \mathrm{~mm}$ continuum emission of G9.62+0.20E, Sanna et al. (2015) suggest that the periodic masers in this source can be accounted for by the presence of an independent pulsating young massive star within the context as proposed by Inayoshi et al. (2013). In addition to these two papers, Szymczak et al. (2015) recently presented the discovery of four new periodic masers and an updated light curve for $\mathrm{G} 22.357+0.066$ which is strikingly similar to that of the $12.2 \mathrm{GHz}$ masers in G9.62+0.20E. These authors argue that although the colliding-wind binary (CWB) model of van der Walt (2011) can explain the flare profile of G22.357+0.066, models in which the maser flares are related to changes in the maser optical depths and excitation temperatures - such as that of Inayoshi et al. (2013) and Parfenov \& Sobolev (2014) - are preferred to the CWB explanation.

Given that the flaring behaviour of the $12.2 \mathrm{GHz}$ masers in $\mathrm{G} 9.62+0.20 \mathrm{E}$ and the $6.7 \mathrm{GHz}$ masers in $\mathrm{G} 22.357+0.066$ are so similar, it can be expected that the physical mechanism underlying the maser flaring is the same in these two sources. Inspection of the light curves presented by Szymczak et al. (2015) rather strongly suggests that the flaring behaviour of the masers in G45.473+0.134 has the same characteristics of that seen in G9.62+0.20E and G22.357+0.066. Thus, at least for these three sources, we are faced with the question: given the current available data, which of the proposed mechanims, i.e. a colliding-wind binary, a pulsating young high-mass star, or rotating spiral shocks in a disk seen edge on (according to Parfenov \& Sobolev 2014), is the most probable mechanism to explain the maser flaring?

In this note we argue on the basis of the expected maser flare profiles associated with a pulsating star driven by the $\kappa$ mechanism, that the proposal by Sanna et al. (2015) cannot explain the observed light curves in G9.62+0.20E, and therefore, by implication, nor those of G22.357+0.066 and G45.473+0.134. We also present an analysis of the model of Parfenov \& Sobolev (2014) and argue that this model cannot explain the flaring behaviour of the masers in the three sources mentioned.

\section{Analysis of models and proposals}

\subsection{Proposal by Sanna et al. (2015)}

The motivation for the proposal by Sanna et al. (2015) that the periodic flaring of the class II methanol masers in G9.62+0.20E is due to a pulsating young high-mass star is the presence of a weaker radio continuum source $1300 \mathrm{AU}$ from the strongest radio continuum component. These authors argue that the stronger radio continuum source is most likely the primary source of the infrared pumping of the $\mathrm{CH}_{3} \mathrm{OH}$ masers. Sanna et al. (2015) consider that the weaker radio continuum source might be a bloated pulsating high-mass protostar which provides the variable infrared emission that underlies the periodic behaviour of the masers. For the pulsating high-mass protostar, Sanna et al. (2015) assume the model of Inayoshi et al. (2013). In this model the protostar becomes pulsationally unstable when the stellar radius expands maximally at a given accretion rate; the instability is caused by the $\kappa$ mechanism in the $\mathrm{He}^{+}$layer. The pulsational unstable state continues until the Kelvin-Helmholtz contraction stage is reached when the stellar surface temperature increases and the $\mathrm{He}^{+}$ionization layer disappears. 
The important point to note here is that within the framework of the model of Inayoshi et al. (2013), the pulsations are driven by the $\kappa$ mechanism, similar to that of other pulsating stars such as the Cepheids. Thus, considering the physics underlying the pulsations, it is reasonable to expect that the luminosity of the young pulsating star should have a light curve similar to that of the Cepheids.

The question now is what the expected light curve for the masers will be if the luminosity of the pulsating star, which heats the surrounding dust and therefore affect the pumping of the masers, varies in a way similar to that of the Cepheids. To address this question we use the $\mathrm{OH}$ masers associated with Mira variables as examples of pulsating stars for which both the optical and maser light curves are known and from which the relation between the $\mathrm{OH}$ maser and optical light curves can be deduced. In this regard, Etoka \& Le Squeren (2000) have presented and compared the behaviour of the $\mathrm{OH}$ masers and optical light curves for seven Mira variables. These authors conclude that although the optical light curves are more strongly asymmetric than that of the associated $\mathrm{OH}$ masers, the shapes of the optical and $\mathrm{OH}$ maser light curves are very similar. This applies to $1612 \mathrm{MHz}, 1665 \mathrm{MHz}$, and $1667 \mathrm{MHz}$ maser transitions even though the degree of saturation does not seem to be the same for all three transitions. Although the example of the Miras might not be exactly equivalent to the scenario envisaged in the proposal of Sanna et al. (2015), it nevertheless strongly suggests that if the optical light curve of the pulsating star in the model of Inayoshi et al. (2013) is similar to that of the Cepheids, then it can be expected that the light curve of the methanol masers should be similar in general.

One of the characteristics of the light curves of the Cepheids is that it never reaches a quiescent state, that is, a state in which the star's luminosity stays constant for a significant fraction of the period (see e.g. Yoachim et al. 2009). This is also the case for $\mathrm{OH}$ masers associated with Miras (Etoka \& Le Squeren 2000) as well as for the $\mathrm{OH}$ masers associated with $\mathrm{OH} / \mathrm{IR}$ stars (Engels et al. 2015). However, for both the $12.2 \mathrm{GHz}$ masers in $\mathrm{G} 9.62+0.20 \mathrm{E}$ and the $6.7 \mathrm{GHz}$ masers in $\mathrm{G} 22.357+0.066$, a well-defined quiescent state can be identified (see Fig. 1 and Szymczak et al. 2015). In the case of G9.62+0.20E, the quiescent state lasts for about 115 days (47\% of the period) and for $\mathrm{G} 22.357+0.066$ it is about 65 days ( $37 \%$ of the period). Should the flaring of the methanol masers in G9.62+0.20E be due to the effect of a pulsating star driven by the $\kappa$ mechanism, then the quiescent state of the maser would imply that the star has settled into an equilibrium state for a significant fraction of the period. The physics of the $\kappa$ mechanism, however, does not allow the star to reach equilibrium after each flare and to spontaneously start to pulsate again. Within the framework of the model of Inayoshi et al. (2013), it would mean that after a flare the $\mathrm{He}^{+}$ionization layer disappears but spontaneously reappears at the end of the quiescent phase at which time there should also be a mechanism that trigger the pulsation of the star.

Considering the above, we conclude that the behaviour of the periodic methanol masers in G9.62+0.20E is inconsistent with what is expected if flaring of the masers is driven by a pulsating young high-mass star within the framework of the model of Inayoshi et al. (2013).

\subsection{Model of Parfenov \& Sobolev (2014)}

The Parfenov \& Sobolev (2014) model is significantly more complicated than that of a pulsating star; furthermore there is no direct analogue for this scenario similar to what the Mira
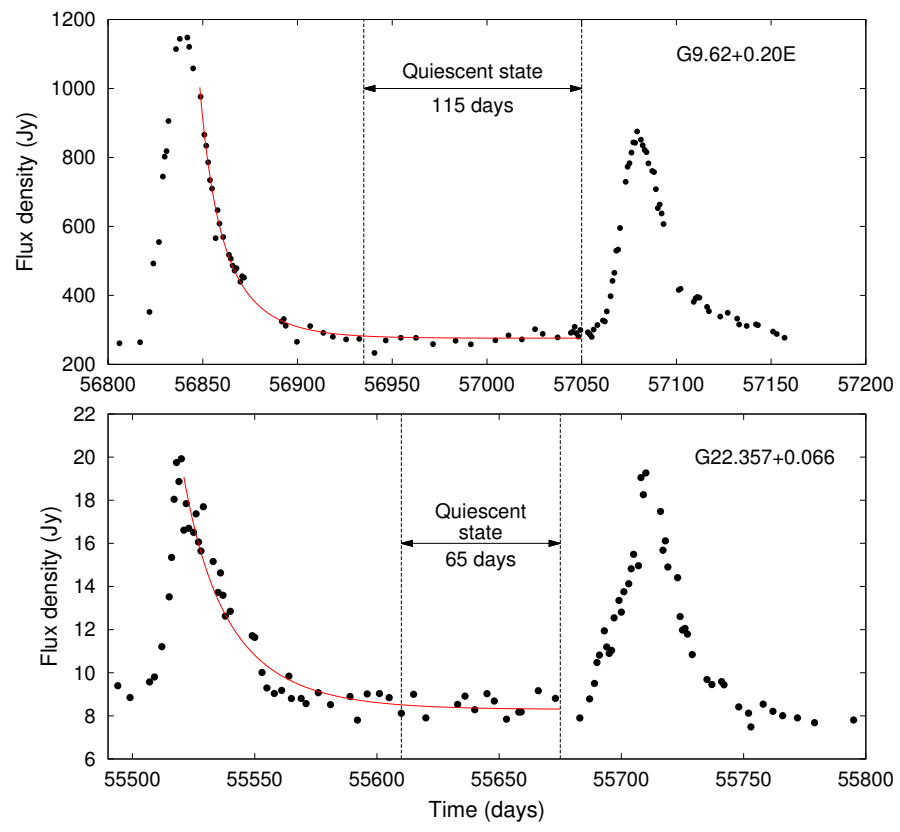

Fig. 1. Selected parts of the timeseries for the $1.25 \mathrm{~km} \mathrm{~s}^{-1}$ feature at $12.2 \mathrm{GHz}$ for $\mathrm{G} 9.62+0.20 \mathrm{E}$ (top panel) and the $80.09 \mathrm{~km} \mathrm{~s}^{-1}$ at $6.7 \mathrm{GHz}$ for $\mathrm{G} 22.357+0.066$. The red solid line in each of the panels is a fit to the decay parts of Eq. (A.7) of van der Walt et al. (2009). The interval of the quiescent state was estimated by eye.

variables and $\mathrm{OH} / \mathrm{IR}$ stars are for the proposal by Inayoshi et al. (2013). A longer discussion is therefore needed. We first present a brief outline of the model of Parfenov \& Sobolev (2014). Based on the information in the paper by Parfenov \& Sobolev (2014), we constructed a geometric model which is used to calculate an expected light curve. We also comment on the properties of the bow shocks as used by Parfenov \& Sobolev (2014) since it has direct implications for the predictions of their model. Finally, we consider the effect that the presence of a stellar wind associated with the more massive star of the binary system might have on the locations and luminosities of the bow shocks. Such a consideration is necessary since it also has direct implications for the predictions of the model by Parfenov \& Sobolev (2014).

\subsubsection{Brief description of the model}

The ideas in the model of Parfenov \& Sobolev (2014) are to a large extent based on the scenarios described by, for example, Ochi et al. (2005) and Sytov et al. (2011), although use is also made of the results by Sytov et al. (2009). Sytov et al. (2011), Kaigorodov et al. (2010), and Fateeva et al. (2011) have run numerical simulations to determine the structure of circumbinary envelopes and the flow of gas in young binary systems. The binary systems considered by these authors consist of lower mass and/or T-Tauri stars. These calculations show the creation of a central region of low density (referred to as the central gap) through the action of bow shocks that form in front of the circumstellar accretions disks of the two stars in the case when the orbital speed of the stars is supersonic. Associated with the bow shocks are also trailing spiral shocks that extend from the outer edges of the circumstellar disks to the inner edge of the circumbinary disk.

Parfenov \& Sobolev (2014) transferred some of the elements of this scenario into their model. The basic ingredient is also a binary system inside the gap region of a flat circumbinary disk. The gap region is circular with a radius of 1.9 AU. The two stars 


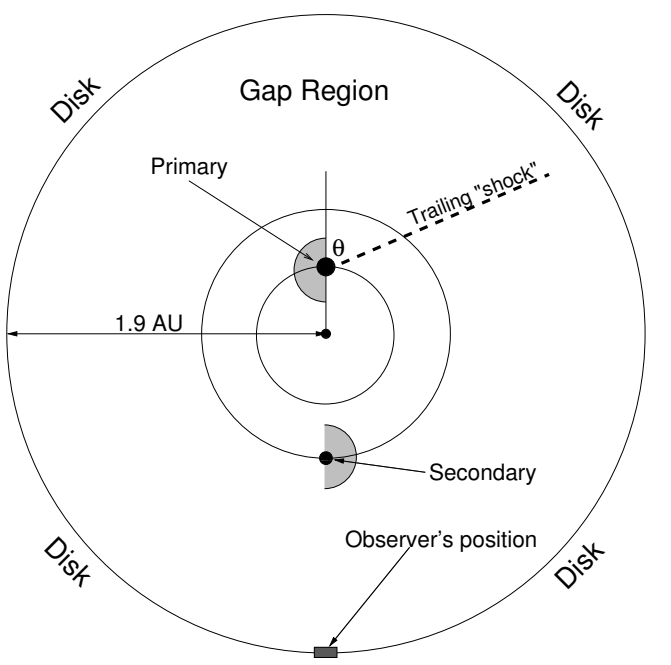

Fig. 2. Schematic representation of the model of Parfenov \& Sobolev (2014). See text for more details. The orbital motion is in an anticlockwise direction.

have masses of $13 M_{\odot}$ (the primary) and $7 M_{\odot}$ (the secondary), respectively. The semimajor axis of the circular orbit is $1.145 \mathrm{AU}$ with a period of 100 days. Using the masses given above, the $13 M_{\odot}$ star follows a circular orbit with radius $0.4 \mathrm{AU}$ around the centre of mass while for the $7 M_{\odot}$ star the radius of its orbit is $0.74 \mathrm{AU}$. The orbital velocity is then found to be $43.6 \mathrm{~km} \mathrm{~s}^{-1}$ for the primary star and $81 \mathrm{~km} \mathrm{~s}^{-1}$ for the secondary star. Parfenov $\&$ Sobolev (2014) do not make reference to the orbital motion of the secondary and it is not clear why, since both stars follow circular orbits around the centre of mass. Within the description given by Parfenov \& Sobolev (2014), a bow shock (to which they also refer as a spiral shock wave) is associated only with the primary star. The material behind the bow shock is dense and hot, resulting in strong UV radiation that can heat the gas and dust on the inner edge of the circumbinary disk. The rotation of this shock is considered to be the main cause for changes in the dust temperature in the circumbinary disk and therefore for the periodic flaring of the masers located in the circumbinary disk. Parfenov \& Sobolev (2014) speculates that the maser brightness traces the change in gas column density along the line between the centre of the disk and a point on the inner edge of the circumbinary disk. This change in gas column density will then give rise to a rather sharp increase in the maser brightness followed by a slow decay, thus explaining the observed light curve of the periodic methanol masers in sources like G9.62+0.20E. In their modelling, the shock is modelled as a half disk with an inner radius of $0.022 \mathrm{AU}$ (the radius of the primary) and an outer radius of $0.2 \mathrm{AU}$.

\subsubsection{Simple geometric model}

Following the description given by Parfenov \& Sobolev (2014), we constructed a schematic representation of their model (Fig. 2). Parfenov \& Sobolev (2014) use the terms "bow shock" and "spiral shock" interchangeably. Here we use the term bow shock only for the shock that forms ahead of the star. We also represent bow shocks with half disks, although in our case the leading edges of the bow shocks are in the instantaneous directions of the velocity vectors of the two stars and not perpendicular to the velocity vector as shown in Fig. $1 \mathrm{~b}$ of Parfenov \& Sobolev (2014). As shown in Fig. 2, a bow shock should be associated with the secondary since its orbital velocity is also supersonic. In this regard, we point to the results of

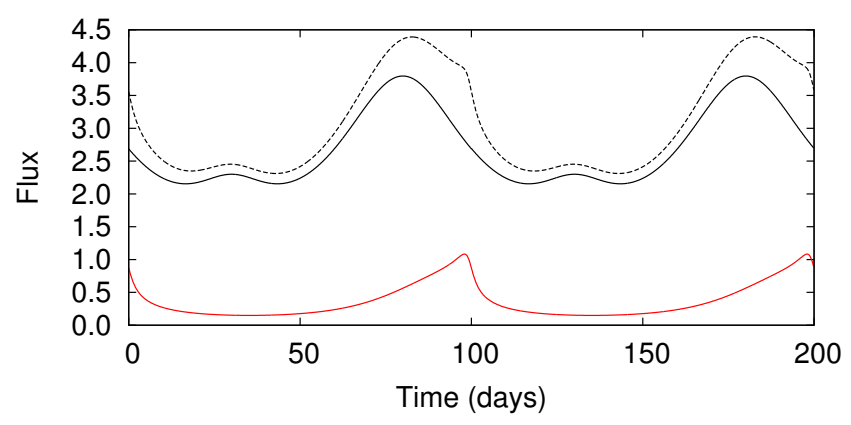

Fig. 3. Expected time dependence of the illumination of a specific point on the inner edge of the circumbinary disk for the cases where the two stars only are considered (solid black line) and for the trailing shock (red line). The values of the parameters used for the trailing shock are given in the text. The dashed black line is the sum of the two cases.

Kaigorodov et al. (2010) where bow shocks are associated with both stars and the bow shocks form ahead of the circumstellar disks associated with the two stars. In what follows, we therefore use the orientation of the bow shocks as shown in Fig. 2. For simplicity, we consider the trailing spiral shock to have the linear structure shown in Fig. 2. We note that, according to e.g. Kaigorodov et al. (2010), spiral shocks are associated with the circumstellar accretion disks around the primary and the secondary. Parfenov \& Sobolev (2014) make no mention of such circumstellar accretion disks.

\subsubsection{Expected light curve}

We first want to remark on the general shape of the light curve expected from such a binary system. The underlying dynamical mechanism in this scenario is the binary system. The orbital motion of the stars, and any other emitting structures associated with the stars, modulates the radiation that illuminates a particular point on the inner edge of the circumbinary disk (henceforth referred to as the observer). The modulation is purely geometric due to the time-dependent variation of $1 / d^{2}$, where $d$ is the distance between each of the stars and the observer. Considering only the two stars (taken as point sources) it is straightforward to calculate the time dependence of the radiative energy flux at the position of the observer given that the ratio of the luminosity of the primary to the secondary is $8.2: 1$. No optical depth effects were taken into account. The result is shown in Fig. 3 as a solid black line. The larger peak is due to the primary star. The effect of the secondary is significantly less because its luminosity is only about $12 \%$ of that of the primary.

It is also rather simple to calculate the effect of the spiral shock if it is approximated as a trailing linear structure as shown in Fig. 2. It can be expected that the volume emissivity of the gas decreases in some way along the length of the shock. To account for this behaviour, we assumed the volume emissivity to decrease as $\left(r / r_{\mathrm{p}}\right)^{-q}$, where $r$ is the radial distance from the disk centre of a point on the shock and $r_{\mathrm{p}}$ is the orbital radius of the primary star. The spiral shock is then considered to consist of a large number of point sources rotating around the centre of mass. The total luminosity of the shocked gas is set to some fraction, $\alpha$, of the luminosity, $L_{\mathrm{p}}$, of the primary; the luminosity of the $i$ th point source is then $L_{i}=L_{0}\left(r_{i} / r_{\mathrm{p}}\right)^{-q}$. The value of $L_{0}$ is determined from the condition that $\sum_{i} L_{i}=\alpha L_{\mathrm{p}}$.

As an example to illustrate the effect of the linear trailing shock, in Fig. 3 we show the time dependence of the illumination at the position of the observer for the case when $\theta=70^{\circ}$ (see Fig. 2), the end point of the shock has an orbital radius of 1.71 AU, $q=2$, and the total radiative luminosity of the shock 
is equal to the luminosity of the secondary star. In reality, the radiative luminosity of the shocked gas may be significantly lower. It is important to note the shape of the light curve: starting from the first minimum there is a rather slow increase in the flux up to a maximum, followed by a rapid decrease towards a minimum. The slow increase occurs when the shock approaches the observer; the different parts of the shock are at different distances, $d$, from the observer and the maximum contribution from the different parts of the shock to the flux at the observer occurs at different times. The maximum in the light curve occurs when the end point of the shock is on its nearest position to the observer, i.e. on the line connecting the centre of the circumbinary disk with the observer. At that position the distance between the base of shock (using the terminology of Parfenov \& Sobolev 2014) and the observer is already so large that the contribution by the base of the shock to the flux is not significant. As the shock moves further, the flux decreases very rapidly owing to the rapid increase in the distance between the end point of the trailing shock and the observer. It is also seen that for the case considered, the peak in the flux due to the shock is delayed relative to the peak due to the primary. The degree to which it is shifted depends on the angle $\theta$ as defined in Fig. 2. Although we used a linear geometry for the shock, the same asymmetric type of light curve is expected for a shock with a spiral structure as in the models of e.g. Kaigorodov et al. (2010), Sytov et al. (2011), and Fateeva et al. (2011).

We have not attempted to calculate the light curve at the position of the observer due to the two bow shocks. Since the presence of the two bow shocks does not significantly break the symmetry for the case when only the two stars are considered, it is expected that the shape of the light curve with the bow shocks included will be rather similar to that of the two stars only.

Now, the case of a binary system, with or without bow or spiral shocks, as the variable source of radiation that heats the dust on the inner edge of the circumbinary disk is in principle no different from that of the Miras or the OH/IR stars discussed earlier. Radiative transfer and reprocessing by dust of the radiation incident on the inner edge of the circumbinary disk up to the region where the masers operate, as well as the response of the masers, will definitely further modify the incident light curve to eventually produce the observed light curve of the masers. Given the example of the Miras, it can be concluded that if the light curve of the variable source at the position of the observer is that shown by the dashed line in Fig. 3, then the masers should have a similar light curve, although not exactly the same. It is therefore difficult to see how a combination of the orbital motion of the stars, the presence of bow or spiral shocks, the normal dust reprocessing of radiation as well as the response of the masers can produce a final maser light curve that resembles the light curve seen for example in the $12.2 \mathrm{GHz}$ methanol masers in G9.62+0.20E (Fig. 1).

Our conclusion here is that the expected light curve of the masers in the binary scenario proposed by Parfenov \& Sobolev (2014) will be quite different from that suggested by these authors and that the observed methanol maser flaring in G9.62+0.20E cannot be explained with this model.

\subsubsection{Comments on the properties of the shocks in the model of Parfenov \& Sobolev (2014)}

In the above analysis, we assumed the bow shocks to have the properties used by Parfenov \& Sobolev (2014). However, it is necessary to note the following: Parfenov \& Sobolev (2014) modelled the bow shock as a half disk of uniform temperature
$(30222 \mathrm{~K})$ and density. Two densities are assumed, $10^{13.32}$ and $10^{13.62} \mathrm{~cm}^{-3}$. The maximum jump in density across a shock is a factor of four in the case of a strong shock (Dopita \& Sutherland 2003; Lequeux 2005). Assuming the strong shock limit to apply, the implication is that the density in the preshocked gas had to be respectively $10^{12.72}$ and $10^{13.02} \mathrm{~cm}^{-3}$ for the above assumed densities of the postshocked gas. However, Parfenov \& Sobolev (2014) assumed a hydrogen density of $10^{9.8} \mathrm{~cm}^{-3}$ at the inner edge of the circumbinary disk, which is significantly lower than the value expected for the preshocked gas assuming the strong shock limit. If the inner edge of the circumbinary disk is to be well defined, then the density of the "low-density hot gas" in the gap region should be lower than $10^{9.8} \mathrm{~cm}^{-3}$. Considering the results of Kaigorodov et al. (2010) and Fateeva et al. (2011), the density in the gap region is at least two orders of magnitude lower than at the inner edge of the circumbinary disk. This would mean that the gas in the gap region should have a density of at most $10^{7.8} \mathrm{~cm}^{-3}$ and the postshocked gas in the bow shock a density of $10^{8.4} \mathrm{~cm}^{-3}$, i.e. at least five orders of magnitude lower than that used by Parfenov \& Sobolev (2014). The implications of such a low value for the density of the postshocked gas is obviously that the luminosity of the shocks will be significantly lower than estimated by Parfenov \& Sobolev (2014).

\subsubsection{Effect of stellar winds}

There is one more aspect related to bow shocks that should be considered. Although Parfenov \& Sobolev (2014) make no mention of stellar winds, it is reasonable to assume, from the given properties of the stars, that both stars have stellar winds. Using Fig. 4 of Kudritzki \& Puls (2000), the wind speed for a main-sequence star with $T_{\text {eff }}=20000 \mathrm{~K}$ is of the order of a few $100 \mathrm{~km} \mathrm{~s}^{-1}$. In the case of a main-sequence star with $T_{\text {eff }}=29000 \mathrm{~K}$, it ranges between about $1000 \mathrm{~km} \mathrm{~s}^{-1}$ and about $2300 \mathrm{~km} \mathrm{~s}^{-1}$. For illustrative purposes, we will use wind speeds of $800 \mathrm{~km} \mathrm{~s}^{-1}$ and $1500 \mathrm{~km} \mathrm{~s}^{-1}$ for the secondary and primary stars, respectively. Also associated with the winds is the massloss rate, $\dot{M}$. For this, we use the results of Vink et al. (2000) and take $\dot{M}$ to be $10^{-7.5} M_{\odot} \mathrm{yr}^{-1}$ (their Fig. 2) for the primary. The parameters of the secondary fall outside of the range covered by the calculations of Vink et al. (2000), but we will arbitrarily take $\dot{M}$ as $10^{-8.5} M_{\odot} \mathrm{yr}^{-1}$.

Given the wind velocities and the mass-loss rates, it is now possible to calculate the stand-off distances of the bow shocks and their luminosities due to the supersonic speed of both stars and the interaction of their winds with the low-density gas in the gap region. The stand-off distance is given by Wilkin (1996) as

$R_{0}=\sqrt{\frac{\dot{M} V_{\mathrm{w}}}{4 \pi \rho_{a} V_{\mathrm{s}}^{2}}}$,

where $\rho_{a}$ is the mass density of the low-density gas in the gap region and $V_{\mathrm{w}}$ and $V_{\mathrm{s}}$ are respectively the wind and stellar velocities. Using for $\rho_{a}$ the value corresponding to $10^{7.8} \mathrm{~cm}^{-3}$, we find stand-off distances of 7.3 AU and 0.9 AU for the bow shocks associated with the primary and secondary, respectively. The implication of the value of 7.3 AU for the stand-off distance for the bow shock associated with the primary is that, rather than forming a bow shock, the wind of the primary will directly interact with the inner edge of the circumbinary disk.

The upper limit for the radiative luminosity of a bow shock is given by Wilkin et al. (1997) as

$L_{\text {shock }}=\frac{1}{2} \dot{M}\left(V_{\mathrm{w}}^{2}+V_{\mathrm{s}}^{2}\right)$. 
From this, we find maximum radiative luminosities of $5.92 L_{\odot}$ and $0.17 L_{\odot}$ for the bow shocks associated with the primary and secondary respectively. Both of these are significantly smaller that the luminosities of the associated stars. The implication of these numbers is that the associated bow shocks do not play a significant role in the radiative energy that hits the circumbinary disk's inner boundary and is in contradiction with the results of Parfenov \& Sobolev (2014).

The presence of the winds also implies the interaction of the winds. This means that there is a contact discontinuity between the two stars with shocked wind material on both sides of it. With the wind speeds assumed above, and using Eq. (2) of Parfenov \& Sobolev (2014), the temperature of the shocked wind of the primary will be about $3.6 \times 10^{7} \mathrm{~K}$ and $10^{7} \mathrm{~K}$ for the shocked wind of the secondary. The system proposed by Parfenov \& Sobolev (2014) is therefore actually a colliding-wind binary within the gap of a circumbinary disk. If $d_{1}$ and $d_{2}$ respectively denote the distances to the contact discontinuity of the primary and the secondary, then the ratio $d_{2} / d_{1}$ is given by

$\frac{d_{2}}{d_{1}}=\sqrt{\frac{\dot{M}_{2} V_{w, 2}}{\dot{M}_{1} V_{w, 1}}}$

(Stevens et al. 1992). From this expression it is found that $d_{1}=$ $0.93 \mathrm{AU}$, which means that the contact discontinuity is situated on the same side as the secondary and has a circular orbit with a radius of $0.19 \mathrm{AU}$ around the centre of mass. Although the temperatures of the shocked winds are quite high, the radiative luminosity of the shocked gas is low. Using the wind speed and mass-loss rate for the primary as above, the total mechanical luminosity of the wind is $5.9 L_{\odot}$. If, for example, gas flowing out in $\pi$ steradians are effectively shocked, then the upper limit for the radiative luminosity of the shocked gas of the primary is only about $1.48 L_{\odot}$, which in this case is only $0.01 \%$ of the luminosity of the primary.

\section{Discussion and conclusions}

Although it is necessary to consider all available observational data when trying to explain the periodic masers in a specific source, it cannot be denied that the light curve is certainly one of the most important pieces of observational information that points to the mechanism underlying the periodic behaviour. The case of the $\mathrm{OH}$ masers associated with Mira variables is a real example which illustrates that the light curves of the $\mathrm{OH}$ masers reflect the basic underlying periodic mechanism. The same is expected to apply to all periodic methanol masers. As already argued above, the light curves expected from a pulsating star or from the binary system as proposed by Parfenov \& Sobolev (2014) are completely different from the observed light curve of the periodic methanol masers in G9.62+0.20E. It is therefore unlikely that either of these two mechanisms underlie the periodic flaring of the methanol masers in sources such as G9.62+0.20E.

In the upper panel of Fig. 1, we also show the fit of equation A7 of van der Walt et al. (2009) to the decay part of the first of the two shown flares. Considering the quality of the fit over an interval of 200 days, it seems reasonable to conclude that the flaring of the methanol masers in G9.62+0.20E is likely to be due to the maser reflecting changes in the flux of free-free emission from a background thermal hydrogen plasma being excited from an equilibrium state of ionization (as set by the presence of the high-mass star) to a higher state of ionization, followed by recombination to the level set by the equilibrium state. There must, however, be a mechanism to explain the periodic increase of the ionization level of that part of the H II against which the maser is projected. Whether the underlying driving mechanism is a colliding-wind binary system has to be verified with other observations, e.g. the presence of associated X-ray emission.

To what extent there are other mechanisms that can equally well describe the maser flare profile in G9.62+0.20E (and sources with similar maser flare profiles) is not clear since periodic variability can only be associated either with the young star or with a binary system. From our discussion above it seems, however, that different physical mechanisms used to explain the periodicity will give rise to different flare profiles. Having ruled out the possibility that it can be due to the pulsation of the young star, it follows that the methanol maser flare profiles in G9.62+0.20E (and other sources with similar flare profiles) are most probably due to some periodic process associated with a binary system that affects the ionization level of parts of the background H II region.

Although our discussion above is focused on the periodic masers in G9.62+0.20E, the general requirement is that any proposed model for the periodic masers in a particular source must also be able to explain the observed maser light curve. It was noted rather explicitly by Szymczak et al. (2015) that three of their newly detected periodic masers have light curves consistent with the CWB model of van der Walt (2011). Together with $\mathrm{G} 22.357+0.066$, this brings the number of periodic masers with light curves similar to G9.62+0.20E to five. Inspection of the light curves of the remaining nine periodic maser sources shows that the majority has the characteristic of not showing a quiescent state. Whether the periodicity in these sources is driven by a pulsating young star is not clear. Since there is no explicit example of a pulsating young massive star it cannot be excluded that there might be pulsation mechanisms other than that proposed by Inayoshi et al. (2013), which might be able to explain the light curves of periodic methanol masers that cannot be explained within the framework of the colliding-wind binary model.

Acknowledgements. This work was supported by the National Research Foundation under Grant number 2053475.

\section{References}

Dopita, M. A., \& Sutherland, R. S. 2003, Astrophysics of the diffuse universe (Springer)

Engels, D., Etoka, S., Gérard, E., \& Richards, A. 2015, in ASP Conf. Ser. 497, eds. F. Kerschbaum, R. F. Wing, \& J. Hron, 473

Etoka, S., \& Le Squeren, A. M. 2000, A\&AS, 146, 179

Fateeva, A. M., Bisikalo, D. V., Kaygorodov, P. V., \& Sytov, A. Y. 2011, Ap\&SS, 335,125

Inayoshi, K., Sugiyama, K., Hosokawa, T., Motogi, K., \& Tanaka, K. E. I. 2013, ApJ, 769, L20

Kaigorodov, P. V., Bisikalo, D. V., Fateeva, A. M., \& Sytov, A. Y. 2010, Astron. Rep., 54, 1078

Kudritzki, R.-P., \& Puls, J. 2000, ARA\&A, 38, 613

Lequeux, J. 2005, The Interstellar Medium (Berlin: Springer)

Ochi, Y., Sugimoto, K., \& Hanawa, T. 2005, ApJ, 623, 922

Parfenov, S. Y., \& Sobolev, A. M. 2014, MNRAS, 444, 620

Sanna, A., Menten, K. M., Carrasco-González, C., et al. 2015, ApJ, 804, L2

Stevens, I. R., Blondin, J. M., \& Pollock, A. M. T. 1992, ApJ, 386, 265

Sytov, A. Y., Bisikalo, D. V., Kaigorodov, P. V., \& Boyarchuk, A. A. 2009, Astron. Rep., 53, 428

Sytov, A. Y., Kaigorodov, P. V., Fateeva, A. M., \& Bisikalo, D. V. 2011, Astron. Rep., 55, 793

Szymczak, M., Wolak, P., \& Bartkiewicz, A. 2015, MNRAS, 448, 2284

van der Walt, D. J. 2011, AJ, 141, 152

van der Walt, D. J., Goedhart, S., \& Gaylard, M. J. 2009, MNRAS, 398, 961

Vink, J. S., de Koter, A., \& Lamers, H. J. G. L. M. 2000, A\&A, 362, 295

Wilkin, F. P. 1996, ApJ, 459, L31

Wilkin, F. P., Canto, J., \& Raga, A. C. 1997, in Herbig-Haro Flows and the Birth of Stars, eds. B. Reipurth, \& C. Bertout, IAU Symp., 182, 343

Yoachim, P., McCommas, L. P., Dalcanton, J. J., \& Williams, B. F. 2009, AJ, 137, 4697 